\title{
QUALIDADE DO MAMÃO CV. SOLO SUBMETIDO AO CHOQUE TÉRMICO E TRATAMENTO QUARENTENÁRIO POR RADIAÇÃO GAMA ${ }^{1}$
}

\author{
RODRIGO MEIRELLES DE AZEVEDO PIMENTEL ${ }^{2}$, YVENS ELY DE MATTOS MARCONDES 3 , \\ JULIO MARCOS MELGES WALDER ${ }^{4}$
}

RESUMO- Para se exportar, é necessário não só atingir padrões de qualidade exigidos pelo país importador, mas também padrões fitossanitários. A irradiação tem como principal função o controle quarentenário de moscas-das-frutas, portanto é necessário que se associe a tratamentos que visem à manutenção da qualidade do fruto. $\mathrm{O}$ objetivo deste trabalho foi avaliar a qualidade dos mamões submetidos à imersão em água quente seguidos de irradiação em doses de tratamento quarentenário. Os mamões verdes selecionados foram submetidos aos seguintes tratamentos: a) controle; b) $250 \mathrm{~Gy}$; c) $500 \mathrm{~Gy}$; d) choque térmico ( $60^{\circ} \mathrm{C}$ por 30 segundos $)$; e) $250 \mathrm{~Gy}$ + choque térmico, e f) $500 \mathrm{~Gy}+$ choque térmico. Os mamões foram armazenados a $21 \pm 1^{\circ} \mathrm{C}$ e umidade relativa de $85-90 \%$, e, após 8 dias, foram avaliados quanto ao teor de sólidos solúveis, $\mathrm{pH}$, acidez titulável, firmeza, índice de doenças e coloração interna. As análises de perda de massa e coloração externa foram realizadas a $0 ; 2 ; 4$ e 7 dias após a irradiação. Foi observado nos frutos submetidos a choque térmico (d, e, f) quantidade menor de sintomas de antracnose e podridão peduncular e, nos tratamentos combinados (e, f), foi verificada manutenção da firmeza do mamão. Somente no segundo dia os frutos irradiados (b, c, e, f) estavam mais amarelados que os demais. Para os demais parâmetros avaliados, não houve diferença entre os tratamentos.

Termos para indexação: papaia, irradiação, pós-colheita, quarentena, choque de calor.

\section{QUALITY OF PAPAYA CV. SOLO SUBMITTED TO HEAT SHOCK AND QUARANTINE TREATMENT BY GAMMA RADIATION}

\begin{abstract}
To export, it is not only necessary to achieve quality standards, but also, phytosanitary standards. The main function of irradiation is quarantine control of fruit flies, therefore it is necessary to combine with treatments which focus at keeping fruit quality. The objective of this work was the evaluation of the quality of papaya submitted to irradiation and heat shock treatment through immersion in hot water. Green papayas selected were submitted in the following treatments: a) control, b) $250 \mathrm{~Gy}$, c) $500 \mathrm{~Gy}$; d) heat shock $\left(60^{\circ} \mathrm{C}\right.$ for 30 seconds), e) $250 \mathrm{~Gy}+$ heat shock and f) $500 \mathrm{~Gy}+$ heat shock. The papayas were stored at $21 \pm 1^{\circ} \mathrm{C}$ with relative humidity of $85-90 \%$, and then after 8 days, they were evaluated by their soluble solid content, $\mathrm{pH}$, titratable acidity, firmness, decay index and internal color. The analysis of fresh matter loss and external color were executed at 0,2, 4 and 7 days after irradiation. It was observed that in treatments submitted to hot water $(\mathrm{d}, \mathrm{e}, \mathrm{f})$ decay was controlled and in combined treatments $(\mathrm{e}, \mathrm{f})$ it was verified higher firmness. In the second day, irradiated treatments (b, c, e, and f) were yellower than the others. As to the other factors evaluated, there were no differences between treatments.
\end{abstract}

Index Terms: Irradiation, post harvest, fruit flies, heat treatment, hot-water dip.

\section{INTRODUÇÃO}

O mamão é um fruto tropical bastante apreciado e de grande importância econômica. A produção de mamão no Brasil é de 1,65 milhão de toneladas, que o torna o maior produtor mundial, sendo que apenas uma parcela relativamente pequena é exportada. Mesmo que as exportações do Brasil tenham aumentado desde o ano 2000, o Brasil é somente o terceiro maior exportador mundial do fruto, sendo o México o maior exportador, com números quase três vezes maiores que os do Brasil (FAO, 2006).

O mamão é de natureza frágil, sendo alvo de várias doenças pós-colheita, além de sofrer danos mecânicos devido a sua casca fina. É, também, um fruto que não suporta temperaturas baixas, aumentando as dificuldades em seu armazenamento e, por fim, ainda se faz necessária para entrada em alguns países a comprovação da eficiência do tratamento quarentenário para as moscas-das-frutas (Bleinroth, 1995).

A irradiação de mamões provou ser eficaz para o controle das moscas-das-frutas em doses relativamente baixas (Moy \& Wong, 2002), além de proporcionar manutenção da firmeza dos frutos por um período de tempo maior quando submetido a doses de 750 Gy. Contudo, não se obteve êxito no controle de doenças (Pimentel \& Walder, 2004).

$\mathrm{O}$ tratamento hidrotérmico ou de choque térmico depende da temperatura da água e do tempo de exposição. $\mathrm{O}$ tratamento tradicional, inicialmente usado na pós-colheita desde a década de 50 , foi o de submeter os frutos de mamão papaia à imersão em água a $49^{\circ} \mathrm{C}$, por 20 minutos, com o objetivo de controlar doenças e sem causar danos fisiológicos (Akamine \& Arisumi, 1953). Com

1(Trabalho 174-06). Recebido em : 07-11-2006. Aceito para publicação em : 13-07-2007.

${ }^{2}$ Doutor em Ciências - CENA/USP azevedopimentel@uol.com.br.

${ }^{3}$ Engenheiro de Alimentos- yvensely@yahoo.com.br.

${ }^{4}$ Professor Associado - CENA/USP Laboratório de Radioentomologia e Irradiação de alimentos jmwalder@cena.usp.br. 
o tempo, ajustes da tecnologia para aplicação a outros frutos determinaram temperaturas que variam de 46 a $60^{\circ} \mathrm{C}$ com tempos de exposição variáveis de 30 segundos a até 10 minutos (BarkaiGolan \& Philips, 1991).

Tratamento quarentenário com água quente para controle de moscas-das-frutas exige exposição do mamão por mais tempo em temperaturas altas, de forma que a cavidade interna do mamão atinja $46,1^{\circ} \mathrm{C}$; para isso, é utilizada a dupla imersão dos frutos em água quente, iniciando com $42^{\circ} \mathrm{C}$ por 30 minutos, seguido de $49^{\circ} \mathrm{C}$, por 20 minutos (Couey \& Reyes, 1986). Esse tratamento apresentou eficiência semelhante ao tratamento tradicional no controle de doenças (Nishijima et al., 1992), porém os frutos têm apresentado qualidade oganoléptica inferior, caracterizada por textura desigual no interior do fruto (Moy \& Wong, 2002).

$\mathrm{O}$ tratamento combinado consiste na aplicação de calor seguido de irradiação, que resulta num efeito sinérgico no controle de doenças do mamão. Combinações eficazes foram relatadas por Silva (1988), cuja aplicação do tratamento térmico de $48^{\circ} \mathrm{C}$, por 20 minutos, e $50^{\circ} \mathrm{C}$, por 10 ou 7,5 minutos, associados a 750 ou 1.000 Gy, controlaram doenças, mas não causaram danos aos mamões.

As moscas-das-frutas são consideradas a principal praga quarentenária do mamão. Para se exportar mamão para áreas livres da praga, é preciso que se certifique que a fruta não esteja, levando-a para o país importador. Um dos tratamentos bastante eficiente é a irradiação, que foi aceito como tratamento quarentenário pelos Estados Unidos no controle da mosca-domediterrâneo (Ceratitis capitata (Wiedemann, 1824)), comum no Brasil, sendo recomendada a dose de $225 \mathrm{~Gy}$. A irradiação aplicada comercialmente é geralmente realizada com o produto embalado e em grandes volumes devido à capacidade de penetração da radiação. Porém, quanto maior o volume, maior a diferença da dose absorvida, sendo que, no centro do contêiner, a dose absorvida é menor e, nas partes mais externas, é maior. Essa variação de dose pode ser de 2 vezes ou mais. Portanto, para o tratamento quarentenário da mosca-do-mediterrâneo, a dose mínima deve ser de $225 \mathrm{~Gy}$, mas, com isso, frutos nas partes mais externas estarão recebendo 450 Gy ou mais (USDA, 2002).

Este trabalho teve por objetivo avaliar a qualidade do mamão submetido ao choque térmico e ao tratamento quarentenário das moscas-das-frutas por irradiação.

\section{MATERIAL E MÉTODOS}

Mamões foram colhidos em 20-09-2006 no sítio "Irmãos Ferrari”, Boa Esperança-ES, no dia anterior à irradiação. Os frutos foram retirados na CEASA - Campinas-SP, e transportados para o Laboratório de Irradiação de Alimentos do Centro de Energia Nuclear na Agricultura-CENA, localizado na cidade de PiracicabaSP. No laboratório, os frutos foram selecionados ainda verdes com apenas uma a duas estrias amarelas e distribuídos ao acaso de acordo com os tratamentos. O experimento foi delineado de forma inteiramente casualizada, num fatorial ( $3 \times 2)$, determinado pelos seguintes tratamentos: sem e com tratamento hidrotérmico a $60^{\circ} \mathrm{C}$, por 30 segundos, e três doses de irradiação $0 ; 250$ e 500 kGy, perfazendo 6 tratamentos, com 4 repetições e 3 frutos por repetição, totalizando 72 frutos.
Primeiramente, realizou-se o tratamento hidrotérmico com água quente em uma caixa com capacidade de 130 litros, acoplandose um aquecedor de água do tipo GTR 190; 1,05 kW na parede da caixa. Com no máximo duas horas após esse tratamento, os frutos foram submetidos à radiação gama, utilizando-se do irradiador Gammabeam 650 (Atomic Energy of Canadá Ltd.).

Após os tratamentos, os frutos foram encaminhados para uma câmara climática onde permaneceram em temperatura de $21 \pm 1^{\circ} \mathrm{C}$ e umidade relativa (UR) do ar em $85-90 \%$, por um período de 8 dias e, ao final, foram realizadas as análises do teor de sólidos solúveis, $\mathrm{pH}$, acidez titulável, firmeza, índice de doenças e coloração interna da polpa dos frutos. As análises de perda de massa fresca e coloração externa foram realizadas em 4 períodos ao longo da armazenagem dos frutos; no dia da irradiação e a 2; 4 e 7 dias após os tratamentos (DAT). Portanto, para esses parâmetros, a análise estatística foi definida por um esquema fatorial $4 \times 3 \times 2$, incluindo o fator período de armazenagem.

Perda de massa fresca: com o auxilio de uma balança digital, as amostras foram avaliadas por diferença entre o peso inicial do fruto no dia do tratamento e o peso final nos dias determinados para avaliação. Os resultados foram expressos em porcentagem de perda de massa.

Coloração externa da casca: a coloração externa da casca do mamão foi determinada por meio de um colorímetro Minolta CR 200b, onde foram feitas três mensurações no fruto ao longo de sua região equatorial, sendo a média dos resultados expressos em ângulo de cor $\left(\mathrm{h}^{\circ}\right)$, de acordo com McGuire (1992).

Índice de doença (ID): a avaliação desse parâmetro foi feita subjetivamente por meio de notas relacionadas com uma escala de categorias adaptada de Azevedo (1998): 0 (zero) referese ao mamão sem nenhum sintoma de doença; 1 refere-se ao mamão com lesões de diâmetro inferior a $1,0 \mathrm{~cm} ; 2$ refere-se ao mamão com lesões de diâmetro entre 1,0 e 2,0 cm; 3 refere-se ao mamão com lesões de diâmetro maior que $2,0 \mathrm{~cm}$. As doenças consideradas na análise foram antracnose e podridão peduncular.

Firmeza: as análises da firmeza da polpa foram realizadas por meio de um penetrômetro manual WAGNER INSTRUMENTS, Force Dial, U.S.A, e expressas em Newtons (N). Foram escolhidos três pontos na região equatorial dos frutos, sendo a média entre eles o valor final.

Cor interna: a cor interna foi determinada utilizando-se do colorímetro Minolta CR 200b. Duas medidas foram tiradas na região equatorial, na parte interna do fruto, após corte longitudinal, sendo os resultados expressos em Croma $\left(\mathrm{C}^{\circ}\right)$, de acordo com McGuire (1992).

Sólidos solúveis: para a avaliação do teor de sólidos solúveis, a polpa extraída do mamão foi diretamente mensurada por um refratômetro Auto Abbe, sendo o resultado expresso em porcentagem de sólidos solúveis.

Acidez titulável: a acidez titulável (AT) foi determinada segundo metodologia proposta por Carvalho et al. (1990), sendo os resultados expressos em porcentagem de ácido cítrico na polpa.

pH: foi avaliado após extração da polpa, por meio de um pHmetro Digimed DMPH-2.

Os dados foram submetidos à analise de variância e, obtendo-se o F significativo ao nível de 5\%, a análise teve continuidade com 
a aplicação do teste de Tukey, com significância a 5\%.

\section{RESULTADOS E DISCUSSÃO}

\section{Perda de massa fresca}

A perda de massa dos mamões não foi influenciada pelos tratamentos estudados. Resultados semelhantes foram apresentados por Pimentel \& Walder (2004) e Miller \& McDonald (1999) onde a irradiação não influenciou na perda de massa. Da mesma forma que o choque térmico, não causou perda de massa em estudo realizado por Oliveira (1999).

Porém, com o passar do tempo de armazenamento, foi verificada perda de massa fresca do mamão (Tabela 1). Essa perda é devido à eliminação natural de água pelos frutos, conforme explica Bleinroth (1992): os frutos, ao longo do seu desenvolvimento, estão quase sempre perdendo água, porém há um suprimento constante pela árvore. Quando se efetua a colheita, permanece a perda de água, mas sem a reposição. A excessiva transpiração compromete a aparência e a textura, tornando-as enrugadas, de coloração mais opaca, murcha, mole e de aspecto "borrachento".

\section{Cor externa}

Ao longo do amadurecimento dos mamões, o ângulo de cor $\left(\mathrm{h}^{\circ}\right)$ foi tornando-se menor, gradativamente, até que atingiu valores que correspondem à cor amarela (Tabela 2).

Segundo relatos de vários autores, a irradiação em mamões pode atrasar(Yasir, 1990), estimular (Ramli \& Yusof, 1992; Pimentel \& Walder, 2004) ou não influenciar o desenvolvimento da cor amarela, sendo que seu principal efeito é a manutenção da firmeza do fruto (Zhao et al., 1996; Moy et al., 1973; Akamine \& Wong, 1966).

No presente experimento, verificou-se que, em frutos irradiados, a mudança da coloração foi mais rápida e, já no segundo dia de armazenamento, estavam mais amarelados que os nãoirradiados. No quarto dia, os não-irradiados apresentavam também a cor amarelada, igualando-se aos irradiados. O desenvolvimento mais rápido da cor pode ser explicado pela alta produção de etileno em decorrência do estresse inicial causado pela irradiação, uma vez que Larrigaudiere et al. (1990), com tomate, e Akamine \& Goo (1971), com mamão, confirmaram a produção significativa de etileno logo após a irradiação desses frutos.

\section{Índice de doenças}

A capacidade de controle de doenças pelos tratamentos combinados não indicou sinergismo entre os tratamentos, ao contrário do que foi relatado por Silva (1988), Akamine \& Wong (1966) e Broderick \& van der Linde (1981), onde o tratamento hidrotérmico aplicado isoladamente foi menos efetivo no controle de doenças do mamão que o tratamento combinado. Contudo, a dose de irradiação utilizada por estes autores foi de $750 \mathrm{~Gy}$, enquanto neste trabalho a maior dose foi de $500 \mathrm{~Gy}$.

Os resultados deste trabalho revelam que o tratamento hidrotérmico, independentemente da aplicação da irradiação, proporcionou melhor controle de doenças no mamão (Tabela 3). Segundo Balbino (1997), a hidrotermia é bastante eficiente no controle de doenças. Por outro lado, a aplicação da irradiação não teve efeito no controle de doenças como também foi verificado por Pimentel \& Walder (2004) e Miller \& McDonald (1999). Moy (1977) relatou que resultados sobre a irradiação de frutos variam por uma série de razões, dentre as quais, um dos itens incluídos foi o grau de infecção inicial do fruto antes da irradiação, que deve ser baixo para o sucesso do tratamento.

\section{Firmeza}

Pelas doses de tratamento quarentenário aplicadas, inferiores às recomendadas para conservação, os resultados revelam que somente quando combinado ao tratamento térmico os mamões irradiados se tornaram significativamente mais firmes que os mamões não-irradiados (Tabela 3 ). A causa provável pode ter sido a maior incidência de doenças nos mamões irradiados em relação aos tratados também por choque térmico. As podridões estimulam a produção de etileno nos frutos que, por conseqüência, acelera o amadurecimento e o amolecimento do mamão (Awad, 1993).

Portanto, o tratamento quarentenário por irradiação combinado ao choque térmico tem uma vantagem comparativa interessante em relação à alternativa de tratamento quarentenário que é a dupla imersão em água quente. Este tratamento consiste na exposição dos mamões em água quente a $41^{\circ} \mathrm{C}$, por 30 minutos, e a $49^{\circ} \mathrm{C}$, por 20 minutos (Couey \& Reyes, 1986), que causa amolecimento e textura desigual no interior do fruto (Moy \& Wong, 2002).

Não foi verificada diferença de firmeza entre mamões submetidos somente a choque térmico e o controle não-tratado (Tabela 3). Oliveira (1999) constatou que o tratamento hidrotérmico de $49^{\circ} \mathrm{C}$, por $20 \mathrm{~min}$, causou amolecimento em mamões e explicou que a perda de firmeza está associada ao aumento das atividades das enzimas poligalacturonase (PG), pectinametilesterase (PME) e b-galactosidase e, como todas as enzimas, são diretamente alteradas com temperaturas elevadas. A PG atua na região mais interna do mesocarpo, e as outras duas, nas mais externas, e, portanto, o tipo de tratamento térmico pode ter alguma relação com o amolecimento do fruto. Sendo assim, tratamentos térmicos mais prolongados atingirão temperaturas altas em profundidade e estimularão por mais tempo as enzimas responsáveis pelo amolecimento. Neste trabalho, não houve amolecimento do fruto porque o tratamento teve uma duração bem menor, de apenas 30 segundos, e o calor não afetou as áreas mais internas do fruto. Akamine \& Wong (1966) também afirmaram que o tratamento com água quente a $49^{\circ} \mathrm{C}$, por 20 minutos, amoleceu o mamão, porém, quando o tratamento de água quente e a irradiação foram aplicados conjuntamente, a firmeza do mamão era mantida em níveis superiores à testemunha e da mesma maneira que ocorria com mamões que somente foram irradiados. É interessante notar que a dose de $500 \mathrm{~Gy}$, precedida pelo tratamento térmico, promoveu maior firmeza ao fruto do que quando aplicada isoladamente (Tabela 3). 
Teor de sólidos solúveis, $\mathrm{pH}$, acidez titulável e cor interna do mamão

Não houve efeito dos tratamentos em estudo nas variáveis: teor de sólidos solúveis, $\mathrm{pH}$, acidez titulável e cor interna do mamão. Esses resultados corroboram os de Moy et al. (1973) e Pimentel \& Walder (2004) que também não verificaram efeito da irradiação nessas variáveis. Em relação ao tratamento hidrotérmico, Albernaz et al. (2003) também não observaram modificações no mamão, nas variáveis: teor de sólidos solúveis, $\mathrm{pH}$, acidez titulável após imersão em água quente, por 20 minutos, a $48^{\circ} \mathrm{C}$.

TABELA 1 - Porcentagem de perda de massa do mamão submetido à irradiação ( 250 e $500 \mathrm{~Gy})$ e choque térmico (CT) com $60^{\circ} \mathrm{C}$,por 30 segundos, em função do período de armazenagem a $21 \pm 1^{\circ} \mathrm{C}$ e UR de $85-90 \%$.

\begin{tabular}{lccccc}
\hline \multirow{5}{*}{ Tratamentos } & \multicolumn{5}{c}{ Dias após tratamento } \\
\cline { 2 - 6 } & 0 & 2 & 4 & 7 & $\mathrm{CV}(\%)$ \\
\hline Controle & $0 \mathrm{Da}$ & $1,41 \mathrm{Ca}$ & $2,05 \mathrm{Ba}$ & $3,43 \mathrm{Aa}$ & 4,51 \\
$250 \mathrm{~Gy}$ & $0 \mathrm{Da}$ & $1,40 \mathrm{Ca}$ & $2,00 \mathrm{Ba}$ & $3,47 \mathrm{Aa}$ & 19,43 \\
$500 \mathrm{~Gy}$ & $0 \mathrm{Da}$ & $1,53 \mathrm{Ca}$ & $2,11 \mathrm{Ba}$ & $3,59 \mathrm{Aa}$ & 5,06 \\
$\mathrm{CT}$ & $0 \mathrm{Da}$ & $1,51 \mathrm{Ca}$ & $2,29 \mathrm{Ba}$ & $3,60 \mathrm{Aa}$ & 9,18 \\
$250 \mathrm{~Gy}+\mathrm{CT}$ & $0 \mathrm{Da}$ & $1,48 \mathrm{Ca}$ & $2,11 \mathrm{Ba}$ & $3,24 \mathrm{Aa}$ & 5,86 \\
$500 \mathrm{~Gy} € \mathrm{~T}$ & $0 \mathrm{Da}$ & $1,35 \mathrm{Ca}$ & $2,01 \mathrm{Ba}$ & $3,23 \mathrm{Aa}$ & 6,45 \\
$\mathrm{CV}(\%)$ & & 6,28 & 6,43 & 8,8 & \\
\hline
\end{tabular}

Valores seguidos por letras distintas (minúsculas nas colunas e maiúsculas nas linhas) foram diferentes, pelo teste de Tukey $(\alpha=0,05)$.

TABELA 2 - Cor externa $\left({ }^{\circ} \mathrm{h}\right)$ do mamão submetido à irradiação (250 e 500Gy) e choque térmico (CT) $\operatorname{com} 60^{\circ} \mathrm{C}$, por 30 segundos, em função do período de armazenagem a $21 \pm 1^{\circ} \mathrm{C}$ e UR de $85-90 \%$.

\begin{tabular}{|c|c|c|c|c|c|}
\hline \multirow[b]{2}{*}{ Tratamentos } & \multicolumn{4}{|c|}{ Dis após tratamento } & \multirow[b]{2}{*}{$\mathrm{CV}(\%)$} \\
\hline & 0 & 2 & 4 & 7 & \\
\hline Controle & $110,15 \mathrm{Aa}$ & $105,52 \mathrm{Aa}$ & $92,01 \mathrm{~B}$ & $83,91 \mathrm{C}$ & 3,32 \\
\hline 250 Gy & $110,84 \mathrm{Aa}$ & $100,74 \mathrm{Bb}$ & $92,24 \mathrm{C}$ & $85,35 \mathrm{D}$ & 2,63 \\
\hline 500 Gy & $107,48 \mathrm{Aa}$ & $97,57 \mathrm{Bb}$ & $87,80 \mathrm{C}$ & $83,64 \mathrm{D}$ & 1,16 \\
\hline $\mathrm{CT}$ & $111,14 \mathrm{Aa}$ & $106,34 \mathrm{Aa}$ & $93,64 \mathrm{~B}$ & $85,44 \mathrm{C}$ & 2,72 \\
\hline $250 \mathrm{~Gy}+\mathrm{CT}$ & $110,07 \mathrm{Aa}$ & $99,92 \mathrm{Bb}$ & $90,48 \mathrm{C}$ & $85,02 \mathrm{D}$ & 1,77 \\
\hline $500 \mathrm{~Gy}+\mathrm{CT}$ & $109,64 \mathrm{Aa}$ & $100,19 \mathrm{Bb}$ & $91,15 \mathrm{C}$ & $85,54 \mathrm{D}$ & 1,48 \\
\hline $\mathrm{CV}(\%)$ & 1,62 & 1,57 & 3,82 & 1,87 & \\
\hline
\end{tabular}

Valores seguidos por letras distintas (minúsculas nas colunas e maiúsculas nas linhas) foram diferentes, pelo teste de Tukey $(\alpha=0,05)$.
TABELA 3 - Índice de doenças (ID) e firmeza dos mamões expresso em Newtons (N), em função do choque térmico e irradiação, após 8 dias de armazenamento, a $21 \pm 1^{\circ} \mathrm{C}$ e UR de $85-90 \%$.

\begin{tabular}{|c|c|c|c|c|}
\hline \multirow[b]{2}{*}{ Choque térmico } & \multicolumn{4}{|c|}{ Irradiação (Gy) } \\
\hline & 0 & 250 & 500 & $\mathrm{CV}(\%)$ \\
\hline \multicolumn{5}{|c|}{ Índice de Doenças } \\
\hline Controle & $2,08 \mathrm{Aa}$ & $2,00 \mathrm{Aa}$ & $2,12 \mathrm{Aa}$ & 22,7 \\
\hline $30 \mathrm{~s} 60 \mathrm{C}$ & $1,00 \mathrm{Ab}$ & $0,58 \mathrm{Ab}$ & $0,81 \mathrm{Ab}$ & 62,02 \\
\hline \multirow[t]{2}{*}{$\mathrm{CV}(\%)$} & 36,21 & 43,23 & 22,43 & \\
\hline & & Firmeza & & \\
\hline Controle & $10,1 \mathrm{Aa}$ & $12,65 \mathrm{Aa}$ & $12,16 \mathrm{Ab}$ & 12,49 \\
\hline $30 \mathrm{~s} 60^{\circ} \mathrm{C}$ & $10,1 \mathrm{Ba}$ & $13,82 \mathrm{Aa}$ & $14,31 \mathrm{Aa}$ & 9,83 \\
\hline $\mathrm{CV}(\%)$ & 13,46 & 11,44 & 8,88 & \\
\hline
\end{tabular}

Valores seguidos por letras distintas (minúsculas nas colunas e maiúsculas nas linhas) foram diferentes, pelo teste de Tukey $(\alpha=0,05)$.

\section{CONCLUSÃO}

A irradiação é uma boa alternativa para tratamento quarentenário, por não alterar negativamente os parâmetros de qualidade dos mamões analisados. Quando associado ao choque térmico, ocorre um menor desenvolvimento das doenças antracnose e podridão peduncular, e os mamões se mantêm mais firmes, demonstrando que há compatibilidade entre os tratamentos.

\section{REFERÊNCIAS}

AKAMINE, E. K.; ARISUMI, T. Control of postharvest storage decay of fruits of papaya (Carica papaya L.) with special reference to the effect of hot water. Proceedings of the American Society for Horticultural Science, Alexandria, v.61, p.270-274, 1953.

AKAMINE, E. K.; GOO, T. Respiration of gamma irradiated fresh fruits. Journal of Food Science, Chicago, v.36, p.1074-1077, 1971. AKAMINE, E.K.; WONG, R.T.E. Extending the shelf life of papayas with gamma irradiation. Hawaiia Farm Science, Honolulu, v.15, n.4, p.4-6, 1966.

ALBERNAZ, D. de F.; MIRANDA, S. de P.; FAGUNDES, G. R.; YAMANISHI, O. K. Efeito do tratamento hidrotérmico na qualidade e vida pós-colheita do mamão (Carica papaya L.), cv. Golden produzido no oeste da Bahia. In: REUNIÃO ANUAL DA SOCIEDADE INTERAMERICANA DE HORTICULTURA TROPICAL, 49., 2003. Fortaleza. Resumos... p.233.

AWAD, M. Fisisologia pós-colheita de frutos. São Paulo: Livraria Nobel, 1993. 114p.

AZEVEDO, L.A.S. Manual de quantificação de doenças de plantas. São Paulo: Novartis Agro, 1998. 114p. 
BALBINO, J. M. de S. Efeitos de hidrotermia, refrigeração e ethefon na qualidade pós-colheita de mamão (Carica papaya $\mathrm{L}$.). 1997. 104 f. Tese (Doutorado) - Universidade Federal de Viçosa, Viçosa, 1997.

BARKAI-GOLAN, R.; PHILLIPS, D. J. Postharvest heat treatment of fresh fruits and vegetables for decay control. Plant Disease, St Paul, v.75, n.11, p.1085-1089, 1991.

BLEINROTH, E.W. Tecnologia pós-colheita de frutas tropicais Campinas: ITAL, 1992. 203p.

BLEINROTH, E.W. Determinação do ponto de colheita. In: BRASIL. Ministério da Agricultura e da Reforma Agrária. Mamão para exportação: procedimentos de Colheita e pós-colheita. Brasília: EMBRAPA-SPI, 1995. p.10-25 (Série Publicações Técnicas FRUPEX, 14).

BRODRICK, H.T.; LINDE, H.J. van der. Technological feasibility studies on combination treatments for subtropical fruits. In: INTERNATIONALATOMIC ENERGY AGENCY. Combination processes in food rrradiation. Vienna: IAEA, 1981. p.141-156.

CARVALHO, C.R.L.; MANTOVANI, D.M.B.; CARVALHO, P.R.N.; MORAES, R.M.M. Análises químicas de alimentos. Campinas: ITAL, 1990. 121p

COUEY, H. M.; HAYES, C. F. Quarantine procedure for Hawaiian papaya using fruit selection and a two-stage hot water immersion. Journal of Economical Entomology, Lanham, v.79, p.1307-1314, 1986.

FOODAND AGRICULTURE ORGANIZATION OF THE UNITED NATIONS. FAOSTAT Agricultural Data. Disponível em: $<\mathrm{http} / /$ /faostat.fao.org/faostat/collections>. Acesso em: 21 set. 2005.

LARRIGAUDIERE, C.; LATCHE, A.; PECH, J.C.; TRIANTAPHYLIDES, C. Short-term effects of $\gamma$-irradiation on 1aminocyclopropane-1-carboxylic acid metabolism in early climacteric cherry tomatoes Plant Physiology, Rockville, v. 92, p.577-581, 1990 .

McGUIRE, R. G. Reporting of objective colour measurements HortScience, Alexandria, v.27, n.12, p. 1254-1255, 1992.

MILLER, W.R.; McDONALD, R.E. Irradiation, stage of maturity at harvest and storage temperature during ripening affect papaya fruit quality. HortScience, Alexandria, v.34, n.6, p.1112-1115, 1999.

MOY, J.H. Potential of gama irradiation of fruits: a review. Journal of Food Technology, Oxford, v.12, p.449-457. 1977.

MOY, J.H.; AKAMINE, E.K.; WENKAN, N.; DOLLAR, A.M.; HANAOKA, M.; KAO, H.Y.; LIU, W.L.; RIVETTI, L.M. Tolerance, quality, and shelf life of gamma irradiated papaya grown in Hawaii, Taiwan and Venezuela. In: INTERNATIONALATOMIC ENERGY
AGENCY. Radiation preservation of food. Vienna: IAEA, 1973. p.375-387.

MOY, J. H.; WONG, L. The efficacy and progress in using radiation as a quarantine treatment of tropical fruits - a case study in Hawaii. Radiation Physics and Chemistry, Gaithersburg, v.63, p.397-401, 2002.

NISHIJIMA, K. A.; MIURA, C. K.; ARMSTRONG, J. W.; BROWN, S. A.; HU, B. K. S. Effect of forced hot-air treatment of papaya fruit on fruit quality and incidence of postharvest diseases. Plant Disease, St Paul, v.78, n.7, p.723-727, 1992.

OLIVEIRA, F. A. de M. B. Comportamento térmico e qualidade pós-colheita do mamão submetido a radiação de microondas e a hidrotermia. 1999. 51f. Dissertação (Mestrado) - Universidade Federal de Viçosa, Viçosa, 1999.

PIMENTEL, R. M. de A.; WALDER, J.M.M. Gamma radiation in papaya harvested at three stages of maturation Scientia Agrícola, Piracicaba, v.61, n.2, p.146-150, 2004

RAMLI, H. bin; YUSOF, N. binti. Effects of irradiation on the organoleptic quality and ripening process of papaya. Jurnal Sains Nuklear Malaysia, Kajang, v.10, n.1-2, p.25-35, 1992.

SILVA, T.M.W. Tratamento térmico e radiação gama no controle de Colletotrichum gloeosporioides (penz.) Penz. Et sacc. Agente causal da antracnose em frutos de mamoeiro (Carica papaya L.). 1988. 155 f. Dissertação (Mestrado) - Escola Superior de Agricultura "Luiz de Queiroz", Universidade de São Paulo, Piracicaba, 1988

UNITED STATES DEPARTMENT OF AGRICULTURE Irradiation Phytosanitary Treatment of Imported Fruits and Vegetables (Final rule) Federal Register, v.67, n.205, 2002. Disponível em: <http:// frwebgate.access.gpo.go/cgi-bin/multidb.cgi>. Acesso em: 1 nov. 2003.

YASIR, M.S. The effect of gamma radiation on the chemical content, texture and shelf life of papaya. Jurnal Sains Nuklear Malaysia, Kajang, v.8, n.1, p.15-23, 1990.

ZHAO, M.; MOY, J.; PAULL, R.E. Effect of gamma-irradiation on ripening papaya pectin. Postharvest Biology and Technology, Washington, v.8, n.3, p.209-222, 1996. 